Message for others My project has highlighted how important it is to involve patients in new initiatives.

For paediatric patients it is really important to understand why parents bring their children in as an emergency.

For change to happen it is important to engage and empower parents to make the right choices for their children.

\section{G593 TEAM POD: A QUALITY IMPROVEMENT SPRINT TO IMPROVE PATHOLOGY SERVICES}

${ }^{1}$ 1S Blackstock, ${ }^{11} \mathrm{R}$ Irvine, ${ }^{22} \mathrm{~F}$ Ejiofor, ${ }^{33} \mathrm{~S}$ Popa, ${ }^{44 \mathrm{~J}}$ Tarma, ${ }^{44} \mathrm{~A}$ Furetta, ${ }^{11} \mathrm{~F}$ Cleugh. ${ }^{1}$ Paediatric Department, St Marys Hospital, Imperial College Healthcare NHS Trust, London, UK; ${ }^{2}$ Quality and Logistics Manager, Pathology Business Services, St Marys Hospital, Imperial Hospital Healthcare NHS Trust, London, UK; ${ }^{3}$ Bioengineering, Imperial College London, London, UK; ${ }^{4}$ Service Design, Royal College of Art, London, UK

\subsection{6/archdischild-2015-308599.542}

Whilst working as trainees in a Paediatric department, it became apparent that there were significant delays in patient pathology results. This impacted on patient care, delaying management decisions and discharges, as well as wasting clinicians' time and hospital resources.

We brought this specific issue to a Quality Improvement Sprint; a 2 day hackathon, which served as a catalyst for change.

Our team of doctors, managers, design students and an engineer (who subsequently became known as Team Pod) performed an onsite analysis of the process from patient bedside to pathology laboratory. The wide range of skill sets and experiences within the team were invaluable for this process. We spent time on the wards, shadowing porters, interviewed staff and using the systems in place, gaining valuable insights into the multifaceted problem.

Many hindrances were highlighted. Equipment on the ward was poorly organised and sample volumes required for various investigations were unclear. Although the hospital had a pneumatic rapid delivery system (Pod System), which took minutes to deliver samples to the laboratory, it was rarely used by ward staff. Most samples were being transferred via a porter, which could take hours. A small portering team covers a vast number of clinical areas. Most pod stations had insufficient or no pods available, and there was a general notion amongst staff that the pod system was 'unreliable.'

Fascinatingly, we discovered that the porters were among the highest users of the pod system. They were gathering samples, then sending them to the laboratories using the pod station in their porters' lodge. Often the porters were even unable to access the laboratories after hours so could not hand-deliver. Furthermore, many of the pods were unlabeled, so laboratory staff did not know which wards to return them to, and therefore sent them to the highest users (A\&E or the Porters) perpetuating the cycle. We calculated that the overall inefficiencies in the blood sampling and transfer process was costing the trust an estimated $£ 1.7$ million per year (time spent finding equipment, waiting for porters, calling up laboratories, repeating tests, and delayed discharges).

Having better identified the nature of the inefficiencies, we formulated a problem statement, and proposed distinct initiatives. This included a re-organisation of equipment on the wards, which we instigated via the ward managers. A paediatric specific reference chart with accurate blood volumes and bottles for tests has been developed. We also approached security services and ensured that all porters had appropriate access to the laboratories during and out of hours. Most significantly, we presented our findings and a business case to the trust pathology

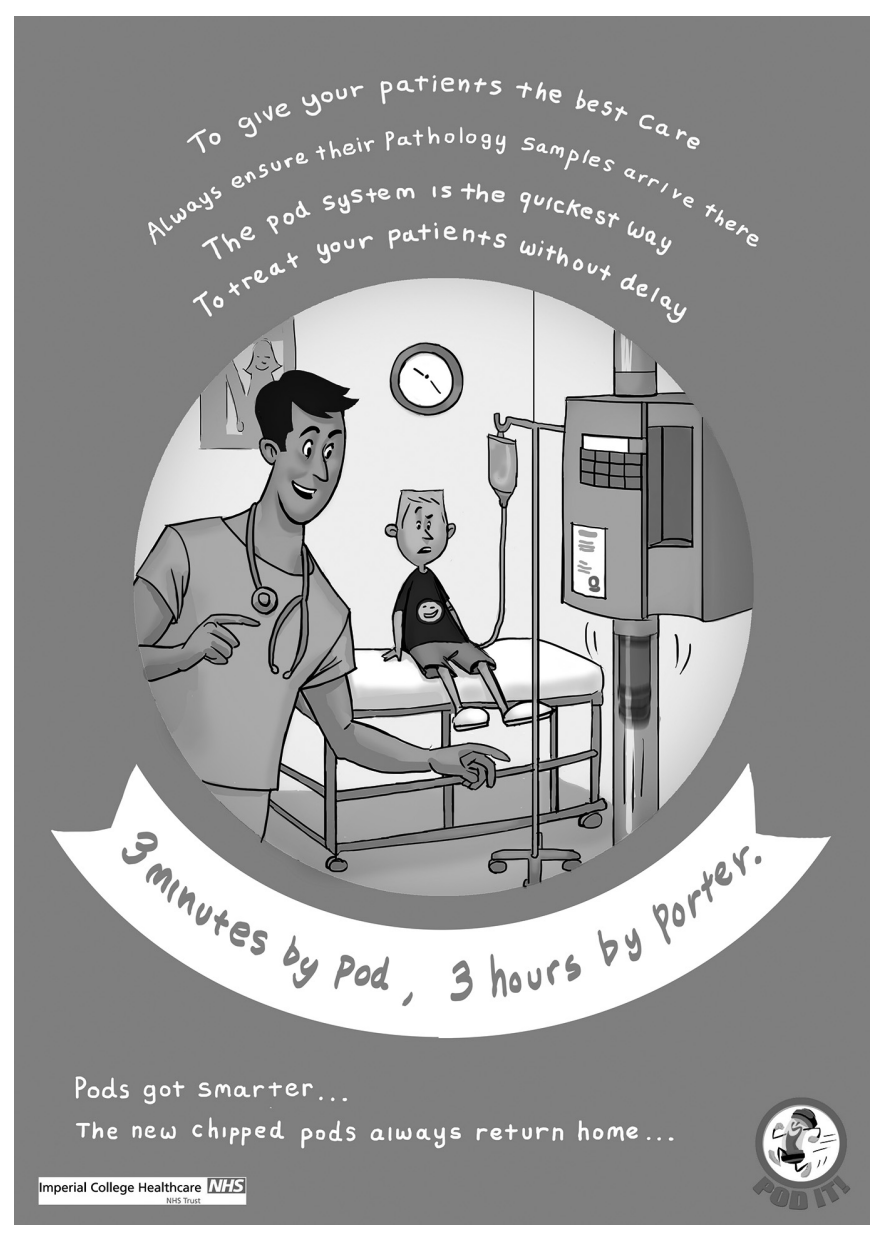

Abstract G593 Figure 1

lead, and a new pod system with trackable 'chipped' pods was approved and implemented. The chipped pods are programmed to always return to their designated station. We then launched a 'pod campaign' to raise awareness of the new system within the trust. The aim was to change the culture and engage staff to realise the benefits and time saved by using the new pod system.

This improvement project demonstrates how basic practices within a hospital can be improved if staff take ownership of the systems they use and engage in efforts to understand and improve processes, and change culture. It was hugely beneficial to have a team with varied experiences and perspectives, and to have dedicated time to focus on a particular issue. Following the two-day hackathon, continued significant efforts were required to see the changes delivered, but the team were motivated and enthused to make a difference. By running these events regularly within NHS trusts, it is possible to mobilise staff to initiate improvements for the care of their patients.

\section{G594 TACKLING HIGH MORTALITY IN A KENYAN DISTRICT HOSPITAL}

B Cahill. Global Links, Royal College of Paediatrics and Child Health, London, UK

\subsection{6/archdischild-2015-308599.543}

Context Completed in a Kenyan hospital paediatric Emergency Department. Clinical officers, Nurses and administration team involved. 


\section{Mortality data in checklist versus non checklist groups}

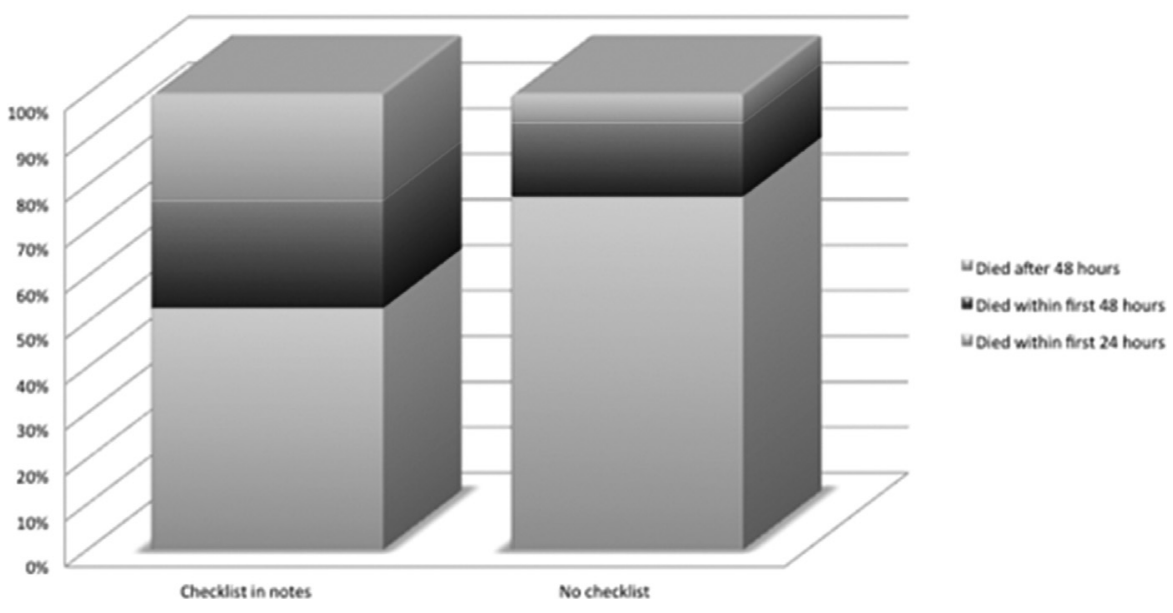

Abstract G594 Figure 1

Problem Stabilisation of paediatric admissions

High mortality levels within first $24 \mathrm{~h}$ of admission.

Often no identifiable cause of death from note keeping.

Lack of documentation of stabilisation procedures.

Clinically unstable children on ward

Assessment of problem and analysis of its causes Liasion with the clinical staff identified lack of full stabilisation of patients prior to ward transfer.

Assessing patient files revealed poor note keeping of stabilisation/lack of early stabilisation interventions.

Intervention Five point checklist designed to standardise early care for all paediatric admissions. Filled out by nurses. The 5 points covered were

- Airway, Breathing and Circulation stable

- Check glucose if not alert

- Take haemoglobin and cross match samples if pale

- Medications written up regulalry on drug chart

- First dose of medications given

The checklist informed staff that each of the points must be completed before the child was to be transferred to the paediatric ward and what to do if these were not performed.

Patient demographics, staff details included.

Study design Retrospective analysis of mortality cases over a 3 month period.

End point examined was change in mortality rates within first $24 \mathrm{~h}$ of admission in intervention and non intervention populations.

Strategy for change 'Safety checklist' designed and trialled by nursing team in the Emergency Department. This was refined and expanded to cover 5 key clinical points.

Several meetings with nursing staff were undertaken. Nurses designated to complete the checklist for new admissions. Local consultant paediatrician agreed to trial of checklist in notes.

From September 2014 administration staff were able to put the checklist in every child's file prior to Emergency Department clerking.

Results of the study have been discussed with the teams involved and both nurses and clinical officers in the emergency department have agreed to continue working with the checklist.

Further data from the hospital has been requested to monitor for ongoing change.
Measurement of improvement All available mortality files over a three month period July-September 2014 were reviewed. Groups with a checklist and without were compared at different time intervals from admission.

All availabel notes of paediatric admissions to the hospital during the same period were reviewed to identify the uptake of the checklist prior to it being put in to all medical files.

A 24\% reduction in mortality within the first $24 \mathrm{~h}$ was observed in the checklist group compared with the non-checklist group.

Effects of changes Initial results reveal that a lower percentage of deaths were in the first $24 \mathrm{~h}$ of admission in the checklist versus non checklist group.

Further data is required to allow month on month comparisons now that every child is receiving a checklist in their notes to see if there is a direct effect on total mortality figures.

Objectively fewer numbers of unstable patients seen on the ward.

There were initial difficulties with getting people to fill in the checklist and insert it in to the patient's file. This was much improved by getting the checklist put in the patient notes by the clinical records team before the child was admitted.

Nurses team nominated for a Kenyan award for their using checklist to improve clinical governance measures.

Lessons learnt To liaise early with nursing team regarding additions to improvement project.

To get one group of staff to take responsibility for checklist completion as avoids confusion of roles.

To get records department to include checklist in admission clerking sheets to allow ease of use and continuity of provision of checklist.

Message for others Simple checklist intervention can have a substantial influence on child mortality within the first $24 \mathrm{~h}$ of admission and lead to better level of standardisation of care for acutely unwell children.

\section{G595 IMPROVING DISCHARGE SUMMARIES-IMPROVING QUALITY OF CARE}

J Stojanovic, S Cornejo Alonso, E Brennan, D Hothi. Great Ormond Street Hospital for Children NHS Foundation Trust, London, UK

10.1136/archdischild-2015-308599.544 